\title{
Acute Physiology and Chronic Health Evaluation Apache II - An ICU Scoring Tool to Assess Critically Ill
}

\author{
Abdul Majeed Arshad ${ }^{1}$, Deepika Ramachandran², Hariharan³, Surya4, Sindhura Koganti ${ }^{5}$, Dhanasekar Thangasamy ${ }^{6}$ \\ B. Rajagopalan ${ }^{7}$
}

${ }^{1}$ Department of Respiratory Medicine, Sri Ramachandra Institute of Higher Education and Research, Chennai, Tamilnadu, India. ${ }^{2}$ Department of Respiratory Medicine, Sri Ramachandra Institute of Higher Education and Research, Chennai, Tamilnadu, India. ${ }^{3}$ Department of Respiratory Medicine, Sri Ramachandra Institute of Higher Education and Research, Chennai, Tamilnadu, India. ${ }^{4}$ Department of Respiratory Medicine, Sri Ramachandra Institute of Higher Education and Research, Chennai, Tamilnadu, India. ${ }^{5}$ Department of Respiratory Medicine, Sri Ramachandra Institute of Higher Education and Research, Chennai, Tamilnadu, India. ${ }^{6}$ Department of Respiratory Medicine, Sri Ramachandra Institute of Higher Education and Research, Chennai, Tamilnadu, India. ${ }^{7}$ Department of Respiratory Medicine, Sri Ramachandra Institute of Higher Education and Research, Chennai, Tamilnadu, India.

\section{ABSTRACT}

\section{BACKGROUND}

The Acute Physiology and Chronic Health Evaluation (APACHE II) is the severity score and mortality estimation tool developed for use in ICU's around the world. It is calculated during the first twenty four hours of admission of the patient to an ICU. An integer score ranges from values 0 to 71 . During the stay, the score is not recalculated; and by definition, is an admission score.

\section{METHODS}

This is a cross sectional study done on 100 patients admitted in the medical ICU in Sri Ramachandra Institute of Higher Education and Research.

\section{RESULTS}

Our results showed an APACHE II score of 16.5 which was significant and values equal to and higher than that correlated with the severity of illness.

\section{CONCLUSIONS}

APACHE II score is a good predictor of mortality in acute respiratory failure patients. It is a useful audit tool which helps the healthcare professionals to interpret and compare their care performance over time.

\section{KEY WORDS}

ICU, Apache, Respiratory Failure, Mortality

\author{
Corresponding Author: \\ Dr. Abdul Majeed Arshad, \\ Old No. 24/New No. 4, \\ $12^{\text {th }}$ Cross Street, Shastri Nagar, \\ Adyar, Chennai-600020, \\ Tamilnadu, India. \\ E-mail: dr.arshad.majeed@gmail.com \\ DOI: $10.14260 /$ jemds $/ 2020 / 8$ \\ Financial or Other Competing Interests: \\ None. \\ How to Cite This Article: \\ Arshad AM, Ramachandran D, Hariharan, \\ et al. Acute physiology and chronic health \\ evaluation apache II - an ICU scoring tool \\ to assess critically ill. J. Evolution Med. Dent. \\ Sci.2020;9(07):383-387, \\ 10.14260/jemds/2020/88
}

Submission 28-09-2019,

Peer Review 22-01-2020,

Acceptance 29-01-2020,

Published 17-02-2020. 


\section{BACKGROUND}

The original APACHE classification system started developing since 1978 as a measure to assess, segregate groups of ICU patients and evaluate their care. Since patients with a wide heterogenous variety of diagnoses and severity of illness are received in the ICU. The most important difference is that all the data regarding the severity and diagnoses are collected early in the course of stay, within the first twenty four hours of admission. In medical research, this has thus been a prospective observational study, not a retrospective review.

The underlying basis of APACHE is that the varied physiologic measurements obtained as a routine on ICU patients contain information on the acute severity of the patient's illness. Therefore, the original APACHE had consisted of an acute physiology score (APS) on the basis of thirty four physiologic variables and chronic health assessment. Chronic health assessment was done based on a separate 4 category scale derived from items previously used to assess chronic health by Rand Health Insurance Study, the Health Interview Survey, and the New York Heart Index. Consensus of a group of experienced ICU clinicians was used to select the 34 APS variables and to specify how to weight derangements in each. Several of the 34 variables are measured only on patients with specific diagnoses or symptoms. Therefore, the variables that were unmeasured in the ICU are not likely to be seriously deranged and could be assumed as normal. This is especially true for measurement during the first day in ICU when a wide variety of physiologic variables are routinely repeated snd measured. The translation of thirty four variables into APS weights has been illustrated for the respiratory rate below. There is a wide range of normality that receives a weight of zero and increase in a nonlinear and non-symmetric manner as patient's breaths per minute varies from the normal range in both directions.

APS weights for respiratory rate (breaths per minute). Respiratory rate 5<6-9 10-11 12-24 25-34 35-49 50< APS weight 42034 (formula) 20. The APS score of the patient is calculated as the sum of the weights for the most deranged value of each variable measured within 24 hours of ICU admission. Initial results with APACHE on the first 600 consecutive ICU admissions at George Washington University Medical Center (GW) were quite promising. These patients were distributed widely across the APS score, ranging from 0 to 50's, with a bell-shaped distribution. The APS was significantly sensitive to the outcome of the patient, being survival or death (Knaus, Zimmerman et al., 1981). It was expected that APS would also be significantly related to the resource costs of treatment in the ICU. The patients who are severely ill receive a more complex therapy and generally take longer to recover. This hypothesis was also strongly supported by the data.

Subsequent research demonstrated that APACHE could be measured in a community hospital with equal precision and predictive validity, but that the community hospital had far different patients in its ICU than did GW (Draper et al, 1981).(1) Most of the community hospital ICU patients were there to be observed closely, not aggressively treated. Their average severity of illness was only seven APS points compared to sixteen at GW Hospital despite their similar medical diagnoses. The APS of APACHE was by far the most significant variable in explaining variation in survival and resource cost of care. This accounted for more than fifty percent of the variation in each dependent variable and its regression coefficient was least affected by inclusion or deletion of a number of clinical and other diagnostic variables (Draper et al., 1981). The APS of APACHE is also sensitive at the lower range of severity of illness. ICU patients who were admitted for monitoring and were at low risk of ever needing aggressive and unique ICU therapy were identified using this (Wagner, Knaus, Draper, and Zimmerman, 1983; Knaus, Draper, and Wagner, 1983). Further research revealed that it could be measured accurately in a number of hospitals and that the severity of illness in the first twenty four hours of admission into the ICU, could accurately predict variations from seven to thirty percent in hospital death rates. (Knaus, Draper et al., 1982). This measure proved quite useful in comparing medical practice and ICU case mix differences between France and the United States (Knaus, Le Gall et at., 1982). It was also demonstrated that the APS was significantly associated with outcome with approximately the same magnitude within a number of specific cardiovascular, neurologic, respiratory, and gastrointestinal diagnoses (Wagner, Knaus, and Draper, 1983).

The APACHE II is a mortality estimation tool and a severity core which has been developed for use in ICU's around the world. The first APACHE model was presented by Knaus et al. in 1981.(2) It is applied within the first twenty four hours of admission of a patient into the ICU. A score ranges from the values 0 up to 71 . This is not calculated again during the stay, and by definition, is an admission score. If the patient is discharged and re-admitted, a new APACHE II score is calculated. There have been many intensive care unit (ICU) scoring systems, along with many new ones that are being developed to achieve a quantitative and an objective description of the assessment of the degree of organ dysfunction and the evaluation of morbidity in ICU patients. Scoring systems such as: APACHE II, III and IV, Sepsis-related Organ Failure Assessment Score (SOFA), Simplified Acute Physiology Score (SAPS), Multiple Organ Dysfunction Score (MODS), Mortality Prediction Model (MPM) and Logistic Organ Dysfunction Score (LODS) have become a necessary tool in describing ICU populations and in explaining differences in mortality.(3) APACHE II is the most widely used scoring system in ICUs all over the world.(4) these scoring systems provide the estimate of mortality risks in ICU patients. These scoring systems help in patient assessment at the time of ICU admission and facilitate treatment planning. Also, they help to compare and evaluate both quantity and quality of care between different healthcare institutes.(5) There are three components to the APACHE-II score. Acute physiology score (APS), which is the largest component of the APACHE-II score is derived from twelve clinical measurements that are acquired within the first 24 hours after admission into the ICU. The most abnormal measurement is generally selected to generate the APS component of the APACHE-II score.(6) If a variable has not been measured, it is assigned zero points. The variables include heart rate, internal temperature, respiratory rate, mean arterial pressure, serum sodium, serum creatinine, serum potassium, arterial $\mathrm{pH}$, oxygenation, white blood cells count, haematocrit and GCS. The next component is age adjustment; 1 to 6 points are added for the patients older than 44 years. The third component is chronic health evaluation. Additional adjustment is done for patients with chronic and 
severe organ failure which involve the lungs, heart, liver, kidneys and the immune system. The Aim and objective was to evaluate the efficacy of APACHE-II score in the critically ill patients with acute respiratory failure.

\section{METHODS}

This is a prospective observational study, which was conducted in Multidisciplinary ICU at SRI Ramachandra Hospital, Porur, Chennai. A total of 100 patients with acute respiratory failure were enrolled in the study. Patients were grouped into five categories: 1. COPD, 2. Pulmonary Oedema, 3. ARDS, 4. Community Acquired Pneumonia 5. Other Conditions (OSA, Bronchiectasis, ILD). All patients aged 18 years or above, who were admitted in the ICU and remained for over twenty four hours, had been included. Patients with an incomplete set of physiological variables, patients post CABG and patients who stayed for less than twenty four hours in the ICU were excluded. Indication for ICU admission, demographic data and the presence of chronic illness had been recorded.

The first twenty four hours after admission, APACHE-II score had been calculated using 12 physiological variables. Points had been allocated to the worst values of each of the variables as per the protocol. Chronic health and age were also assigned points similarly. Total sum of $\mathrm{A}, \mathrm{B}$ and $\mathrm{C}$ components constituted the APACHE-II score. The conscious levels were assessed by the Glasgow coma scale. For patients who were intubated, the score had been calculated by their ability to understand, regardless of their speech. The final outcome and the total length of stay was also recorded. All the data was recorded by the primary investigator on a proforma of APACHE-II score.

\section{Statistical Analysis}

All the categorical variables, including APACHE-II score, the age and the outcome in terms of either discharge or death had been presented by percentages and frequencies; chi-square test had been applied to assess the significance of the association of APACHE-II score and age with the patient's outcome. Pearson's correlation coefficient was applied in determining the correlation of APACHE-II with the age and length of stay in the hospital. P-value of $<0.05$ was considered statistically significant.

\section{RESULTS}

100 patients were included in the study, 55 male and 45 female (Figure 2.). Gupta et al from India also reported that males were significantly more $(\mathrm{p}<0.01)$ than females. Average age of study patients was $58.5+/-14.2$ ranging from 18 to 90 years) (Figure 1.). Patients were categorised as $26 \%$ COPD, 13\% pulmonary oedema, $13 \%$ ARDS, $18 \%$ community acquired pneumonia, (Figure 4) $30 \%$ others which included ILD, Bronchiectasis, Obstructive sleep apnoea. Cut off for Apache II score was taken as 16.5 as we achieved optimal specificity and sensitivity. The Area under the curve on the Receiver operator characteristic curve was 0.756 which was a significant value. (Figure 5).
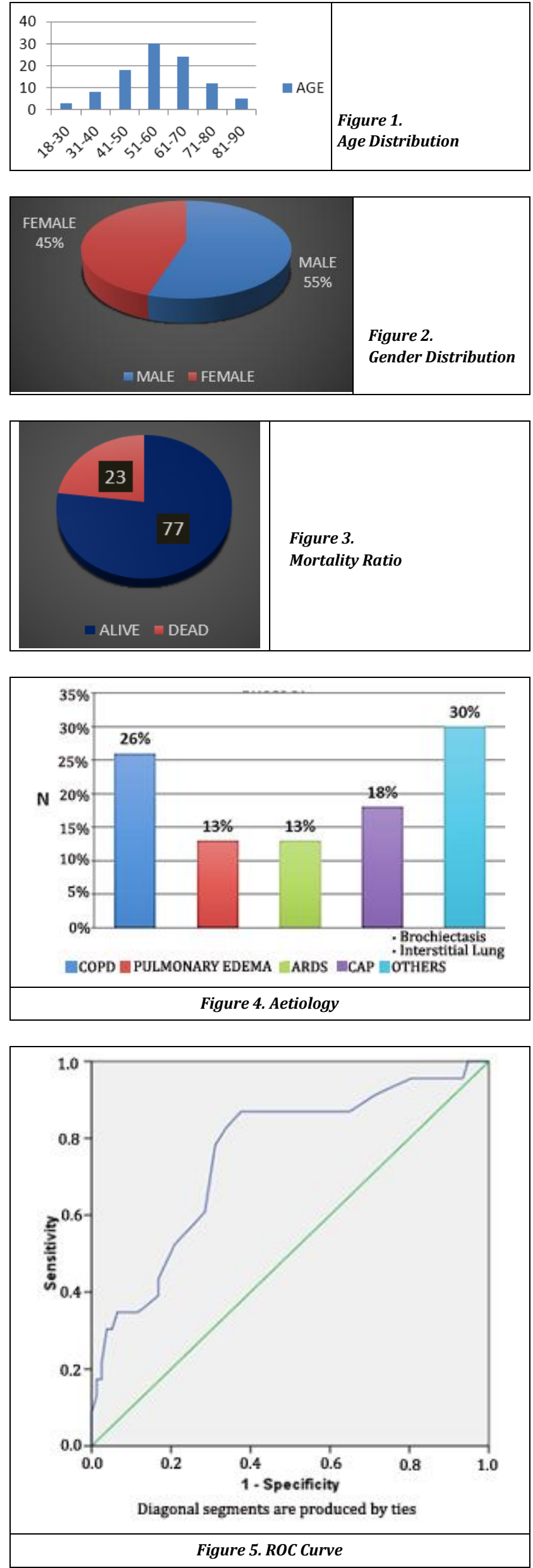
True positives of 20,29 as false positive, false negative of 3 , and true negatives 48 were recorded. The sensitivity was calculated to be $87 \%$, Specificity of $62.3 \%$, Positive predictive value of 40.8 and negative predictive value of $94.1 \%$ Comorbidities such as Diabetes, systemic hypertension, CKD, CHF did not show a positive association with the mortality rate. The physiological variables that showed significance were heart rate and respiratory rate with values ranging from $99+/-18.8$ and $27+/-8$ respectively amongst those alive and $111+/-26$ and $30+/-7$ respectively amongst those dead.

\section{DISCUSSION}

The disparity between demands and available healthcare resources is a universal problem and ICU is an area where this disparity exists up to the maximum, especially in developing countries like India. ${ }^{(7,8)} \mathrm{A}$ critically ill is a person at imminent risk of loss of life and who needs medical treatment in ICU for more than 5 days. At present there are various scoring systems available to predict the outcome of critically ill patients. Although all lack $100 \%$ of accuracy still it plays a vital role to audit the performance outcome of ICU and clinical research. ${ }^{(8)}$ The APACHE II scoring system has been successfully used for predicting the ICU mortality rate in the west by Knaus et al, Wagner et al (1983), Jordan et al, Purdie et al, Marks et al (1991), Brown et al, Van Le et al. However, these trials were from developed countries were the medical facilities are to the optimal level.(9)

Total numbers of 100 patients were included in our study with $55 \%$ of being male and $45 \%$ of being female with mean age of 50.5. Samir Desai et al from Shree Krishna Hospital, Karamsad also reported to have a mean age of 47.52 years which was slightly lower than other studies. Chronological age is a well-documented risk factors for death from acute illness, that is independent of the severity of diseases. Thus age of critically ill patients may have an impact on the severity score. According to literature over $50 \%$ of patients in most ICUs are older than 65 years.(10) In the present study there is no statistically significant difference in mortality compared to gender in ICUs. Mahmood et al from a retrospective review of data among 261,255 patients admitted to adult ICUs in the US had reported that the ICU mortality was $7.9 \%$ for women and $7.2 \%$ for men. Men less than 50 years of age had higher ICU mortality compared to women while 50 years of age or older men had no significant difference compared with women.(11)

It is also reported that diagnosis must be documented within the first day which reflect the primary reason for ICU admission. Perhaps this provides a validation for the health care professionals in taking treatment decisions and to prioritize patient care according to the disease. In our study, the AUC value for APACHE II score is $0.756(\mathrm{p}<0.001)$. The APACHE II scoring system represents a statistically significant predictive marker of fatal outcomes in positivity than negativity. Results of the study reveal that 16.5 could be considered as an acceptable cut-off point for APACHE II score, which could predict death or survival considerable specificity and sensitivity. In this study, the mean APACHE II score is $17.7(\mathrm{SD} \pm 7.75)$. Another similar study conducted by Gupta et al in a respiratory ICU in India had reported 12.87 (SD \pm 8.25 ) for APACHE II. In the same study, it was also mentioned that similar scores had been reported by centres from Saudi Arabia and Singapore. A multi center observational point prevalence study (INDICAPS) conducted in India had patients with moderate severity of illness as evidenced by APACHE II of 17.4(SD \pm 9.2$).{ }^{(12)}$ This signifies that there is a need for improvements in the organization and delivery of critical care in Indian ICUs. APACHE score is declared the "gold standard" for the evaluation of intensive care and is one of the most commonly used scoring system in intensive care unit around the world. It is evident from the previous study that as the APACHE score increases the predicted mortality rate also increases.

The Positive Predicted Value (PPV) (non-survivors) is 40.8 $\%$ and the Negative Predicted Value (NPV) (survivors) is $94.1 \%$. COPD was the leading cause of acute respiratory failure for ICU admission (26 patients, $26 \%$ ), followed by Community Acquired Pneumonia (18 patients, 18\%), Acute Respiratory Distress Syndrome (13 patients, 13\%), Acute Pulmonary Oedema (13 patients, $13 \%$ ) and Other acute respiratory failure conditions like bronchiectasis, bronchial asthma, obstructive sleep apnoea, interstitial lung disease (30 patients, 30\%). Community Acquired Pneumonia (0.017) and ARDS (0.021) showed more significance for APACHE II score in predicting mortality in our study. Thus, considering the dissimilarity which exist among the critically ill admitted to ICUs each intensive care units need to have a mortality prediction system model to validate the patient and to verify with the standard rates. The ability to survive an acute illness can be judged based on the age, gender, comorbidities and the chronic state health disease status.

\section{Importance of This Clinical Research}

The population of patients who are admitted to the ICU is widely varied. Overall outcome of the ICU treatment depends on the age, site, indication of admission, co morbidities and the changes in physiology at the time of admission \& during the initial hours of management. Prediction of mortality of ICU patients in the hospital plays a vital role in assessing the inclusion and exclusion criteria of clinical trials, when we compare predicted mortality with the observed mortality using a scoring system, and in estimating the standardized mortality ratios of the critically ill patients. Need for these predictions has led to the development of various equations in order to calculate the probability of mortality. Though the prognostic scoring systems have been used since the early 1950s (Apgar score for newborns by Virginia Apgar), their application in the assessment of critical patients had only been established in the year 1985 (in which year Knaus et al. had published the second version of APACHE II), which had instantly become the widely applied index for prognosis of Patients in the ICUs worldwide. The predictability of a prognostic index to predict the outcome is assessed based on its discrimination and calibration. Calibration implies correspondence between the expected mortality and the observed mortality in the examined population. The evaluation of this is done by comparing predicted and observed mortality in the mentioned predicted risk groups.(13) The calibration of a prognostic index decreases over time due to the changes in admission and discharge criteria in the ICU, and variations availability and outcome of different methods of management for specific conditions. Thus, developments in intensive medicine over the past three 
decades have caused APACHE II to be rendered obsolete. Presently, this index has caused over-estimation of mortality in a number of scenarios. Further versions of this index, like the recent APACHE IV, at least partially rectify this problem. As described by Soares et al., APACHE II should not be the benchmark tool in ICU because ICUs today are considered high performance on the basis of them having hospital mortality less than expected in the year 1985 .

Discrimination implies ability of the prognostic index in differentiating the patients who die and those who survive. This is assessed by estimating the area under the ROC curve (receiver operating characteristic), (14) with a greater area indicating a better accuracy (as long as it is greater than 0.5). In addition to the varied and heterogeneous patient population in the ICU, one other aspect to consider is the fact that intensive medicine includes syndromes with wide spectra of clinical presentation, like sepsis, delirium, ARDS and postoperative care for surgeries. Therefore, a method is required to assess and categorise the severity of these patients. There is a need for this especially in studies that include a representative population sample to ensure that the findings can be extrapolated to practice. APACHE II was the initial most index to indicate or contraindicate the use of a certain therapy and management, (15) following which, the treatment under review was determined inappropriate. As it continues exhibiting good discrimination capacity, APACHE II stays as a worldwide applied index in describing and assessing the severity in populations of the critically ill.

\section{Limitations}

One constant drawback of APACHE II and its further versions is that these have developed based exclusively on a North American database. This establishes a large region-specific bias in the availability of varied technologies and the modification of patient characteristics to the equations used in these indices cannot correct this bias accurately. In the present day, various other scores are calibrated better and are applied to assess the predicted mortality in order to estimate the severity of patients that are included in the clinical trials.

\section{CONCLUSIONS}

APACHE II score is a good predictor of mortality in acute respiratory failure patients. This study provided an insight into several aspects of critical care in India. Severity scoring systems are used in stratifying critically ill patients to understand the likelihood of the critical care as well as inhospital mortality. It is a useful audit tool which helps the healthcare professionals to interpret and compare their care performance over time. Moreover, it can predict outcome or mortality after discharge and guide in treatment decisions regarding the burden of treatment and success rates of critically ill. It is thereby important for the healthcare professionals to provide comprehensive care for those patients who have higher mortality rates. Furthermore, therapeutic measures can be adopted and evaluated to compare the level of care with international standards and minimize the gaps and bring them closer to the standard values.

\section{REFERENCES}

[1] Draper EA, Wagner DP, Knaus WA. The use of intensive care: a comparison of a university and community hospital. Health Care Financing Review 1981;3(2):49-64.

[2] Knaus WA, Draper EA, Wagner DP, et al. APACHE II: a severity of disease classification system. Crit Care Med 1985;13(10):818-29.

[3] Rapsang AG, Shyam DC. Scoring systems in the intensive care unit: a compendium. Indian J Crit Care Med 2014;18(4):220-8.

[4] Salluh JI, Soares M. ICU severity of illness scores: APACHE, SAPS and MPM. Curr Opin Crit Care 2014;20(5):557-65.

[5] Godinjak A, Iglica A, Rama A, et al. Predictive value of SAPS II and APACHE II scoring system for patient outcome in a MICU. Acta Medica Academica 2016;45(2):97-103.

[6] Lemeshow S, Klar J, Teres D, et al. Mortality probability models for patients in the intensive care unit for 48 or 72 hours: a prospective, multicenter study. Crit Care Med 1994;22(9):1351-8.

[7] Varghese YE, Kalaiselvan MS, Renuka MK, et al. Comparison of acute physiology and chronic health evaluation (APACHE) II and acute physiology and chronic health evaluation(APACHE) IV severity of illness scoring system in a multidisciplinary ICU. J Anaesthesiol Clin Pharmacol 2017;33(2):248-53.

[8] Sekulic AD, Trpkovic SV, Pavlovic AP, et al. Scoring systems in assessing survival of critically III ICU. Patients Med Sci Monit 2015;21:2621-9.

[9] Parajuli BD, Shrestha GS, Pradhan B, et al. Comparison of acute physiology and chronic health evaluation II and acute physiology and chronic health evaluation IV to predict ICU mortality. Indian J Crit Care Med 2015;19(2):87-91.

[10] Gupta R, Arora VK. Performance evaluation of APACHE II score for an Indian patient with respiratory problems. Indian J Med Res 2004;119(6):273-82.

[11] Abdi M, Tofighi S, Niakan-Kalhori S, et al. The quality assessment of performance in intensive care units according to APACHE II Score. Int J Travel Med Glob Health 2015;3(3):125-9.

[12] Divatia JV, Amin PR, Ramakrishnan N, et al. The Intensive Care in India: the Indian intensive care case mix and practice pattern study. Indian J Crit Care Med 2016;20(4):216-25.

[13] Hosmer DW, Lemeshow S. A goodness-of-fit test for the multiple logistic regression model. Commun Stat 1980;9(10):1043-69.

[14] Hanley JA, McNeil BJ. The meaning and use of the area under a receiver operating characteristic (ROC) curve. Radiology 1982;143(1):29-36.

[15] Bernard GR, Vincent JL, Laterre PF, et al. Efficacy and safety of recombinant human activated protein $\mathrm{C}$ for severe sepsis. N Engl J Med 2001;344(10):699-709. 